\title{
ATIVIDADES DE EDUCAÇÃO EM SAÚDE REALIZADAS COM GRUPO DE IDOSAS PARA PROMOÇÃO DO AUTOCUIDADO EM SAÚDE
}

\author{
Francisco Ariclene Oliveira \\ Faculdade Metropolitana da Grande Fortaleza \\ franciscoariclene@hotmail.com \\ Francimara Silva Sousa \\ Faculdade Metropolitana da Grande Fortaleza \\ marinhasousa79@gmail.com \\ Sheyla Lira Cavalcante \\ Faculdade Metropolitana da Grande Fortaleza \\ sheylalira@hotmail.com
}

\author{
Alana Régia Matias Couto \\ Faculdade Metropolitana da Grande Fortaleza \\ alanarmc@hotmail.com \\ Arisa Nara Saldanha de Almeida \\ Faculdade Metropolitana da Grande Fortaleza \\ arisanara@gmail.com \\ Mirian Ferreira Coelho Castelo Branco \\ Faculdade Metropolitana da Grande Fortaleza \\ mirianferreirac@hotmail.com
}

Resumo

Trata-se de um relato de experiência com um grupo de idosas desenvolvido no "Projeto de Extensão e Pesquisa em Saúde da Família - PES" vinculado a uma Instituição de Ensino Superior (IES) de Fortaleza-CE, no período de março a dezembro de 2016. Este artigo objetiva descrever as experiências de atividades de educação em saúde realizadas com idosas tendo como eixos problematizadores Envelhecimento ativo e o Autocuidado. Os encontros foram norteados nos preceitos da Teoria do Autocuidado de Dorothea Orem e nas concepções de educação de Freire. Utilizaram-se ferramentas lúdicas e construtivas, como: oficinas, rodas de conversas, dinâmicas de grupo e recursos audiovisuais, facilitando a reflexão e a construção de saberes entre extensionistas e idosas. O desenvolvimento das atividades possibilitou perceber o quanto essas idosas adquiriram conhecimentos e melhoraram o seu autocuidado. Portanto, urge fortalecer ações preventivas e de promoção da saúde visando promover a prática do autocuidado.

Palavras-chave: Promoção da Saúde. Idosos. Autocuidado.

\section{HEALTH EDUCATION ACTIVITIES CARRIED OUT WITH THE ELDERLY GROUP FOR THE PROMOTION OF SELF-HEALTH CARE IN HEALTH}

\begin{abstract}
This is an experience report with a group of elderly women developed in the "Project for Extension and Research in Family Health" in Fortaleza-CE, in the period from March to December 2016. This article aims to describe the experiences of health education activities carried out with elderly women with the problematic axes: Active Aging and Self Care. The meetings were guided by the precepts of Dorothea Orem's Self-Care Theory and Freire's conceptions of education. It were used playful and constructive tools, such as: workshops, conversation wheels, group dynamics and audiovisual resources, facilitating the reflection and the construction of knowledge between extensionists and the elderly. The development of activities made it possible to perceive how these elderly women acquired knowledge and improved their self-care. Therefore, it is urgent to strengthen preventive and health promotion actions aimed at promoting the practice of self-care. Keywords: Health Promotion. Elderly. Self Care.
\end{abstract}

\section{ACTIVIDADES DE EDUCACIÓN EN SALUD REALIZADAS CON GRUPO DE IDOSAS PARA PROMOCIÓN DEL AUTOCUIDADO EN SALUD}

\footnotetext{
Resumen

Se trata de un relato de experiencia educativa con un grupo de ancianas desarrollado en el "Proyecto de Extensión e Investigación en Salud de la Familia - PES" de Fortaleza-CE, en el período de marzo a diciembre de 2016. Este artículo objetiva describir las experiencias de actividades de educación en salud realizadas com ancianas teniendo como ejes problematizadores el Envejecimiento activo y el Autocuidado. Los encuentros fueron guiados en los preceptos de la Teoría del Autocuidado de Dorothea Orem y en las concepciones de educación de Freire. Se utilizaron herramientas lúdicas y constructivas, como: talleres, ruedas de conversaciones, dinámicas de grupo y recursos audiovisuales, facilitando la reflexión y la construcción de saberes entre extensionistas y ancianas. El desarrollo de las actividades permitió percibir cuánto estas ancianas adquirieron conocimientos y mejoraron su autocuidado. Por lo tanto, urge fortalecer acciones preventivas y de promoción de la salud para promover la práctica del autocuidado.

Palavras clave: Promoción de la Salud. Ancianos. Autocuidado.
} 
Atividades de educação em saúde realizadas com grupo de idosas para promoção do autocuidado em saúde

\section{INTRODUÇÃO}

O Projeto de Extensão e Pesquisa em Saúde da Família - PES, desenvolvido por uma Instituição de Ensino Superior de Fortaleza - IES, destinou-se à formação de grupo de aprendizagem tutorial, favorecendo um elo de parceria entre docentes/discentes de cursos de graduação em áreas da saúde com a comunidade atendida; cumprindo, desse modo, suas atribuições e considerando as Políticas Institucionais de Extensão e Responsabilidade Social nos termos da Resolução no 19 de 27 de setembro de 2012 do CEPEX (FAMETRO, 2012). Nesse sentido, a integração ensino-serviço-comunidade apresenta-se como uma importante estratégia de interação entre universitários, profissionais e usuários despertando um olhar crítico sobre as questões sociais e as potencialidades de um trabalho coletivo para transformação da realidade (SCHOTT, 2016).

O propósito de um desses grupos tutoriais do PES foi promover atividades de educação em saúde a um grupo de idosas vinculadas ao 'Projeto Saúde, Bombeiros de Sociedade' (PSBS) desenvolvido pelo Corpo de Bombeiros Militar do Estado do Ceará (CBMCE), fortalecendo ações preventivas e de promoção da saúde na perspectiva de práticas de educação e saúde, considerando-se o processo de envelhecimento ativo e o autocuidado.

Atualmente no Brasil, os estudiosos da Gerontologia preocupam-se com a forte tendência de envelhecimento da população, caracterizada pelo contingente de pessoas adultas na faixa etária de 40 a 59 anos e que, mesmo considerando as taxas de mortalidade vigente, contribuirão para a expansão do topo da pirâmide populacional nas próximas duas décadas (VALCARENGHI et al., 2015).

Conforme projeções demográficas do Instituto Brasileiro de Geografia e Estatística (IBGE), a população idosa vai triplicar no País e passará de 19,6 milhões (10\% da população brasileira), em 2010, para 66,5 milhões de pessoas, em 2050 (29,3\%). Essas estimativas são de que a "virada" no perfil da população acontecerá em 2030, quando o número absoluto e o porcentual de brasileiros com 60 anos ou mais de idade ultrapassarão o de crianças de 0 a 14 anos (IBGE, 2016).

Dados da Pesquisa Nacional por Amostras de Domicílios (PNAD) constatou que a população idosa do Ceará estava em 1,089 milhões, o que equivale a 12,6\% da população cearense (IBGE, 2015). Esse resultado coloca o Estado como a quinta Unidade da Federação com maior percentual de pessoas com 60 anos ou mais no Brasil. Em relação ao Nordeste, o Ceará fica atrás apenas da Paraíba, que tem $13,1 \%$ da sua população composta por pessoas nesta faixa etária. 
Atividades de educação em saúde realizadas com grupo de idosas para promoção do autocuidado em saúde

O processo de envelhecimento e o consequente aumento da população idosa se devem à redução constante nas taxas de fecundidade e mortalidade, decorrentes da ampliação do sistema de saúde, inclusão de medidas de imunização como, por exemplo, implementação de campanhas vacinais e promoção da qualidade de vida.

Esse processo tem se apresentado em ritmo acelerado com o passar do tempo, consequentemente essa população tem se tornado maior que as demais. Isso é um fator preocupante para a saúde pública devido às características peculiares desse público que necessitam de programas voltados para a promoção do envelhecimento ativo. Requerendo cada vez mais um olhar diferenciado para essa faixa etária, pois o processo de envelhecer, é sem dúvida, algo natural e gradual no ciclo de vida, diferindo de um ser para outro, refletindo uma experiência heterogênea.

Ressalta-se que o envelhecimento pode ser compreendido como um processo sociovital multifacetado que ocorre ao longo de todo o curso da vida (DAWALIBI et al., 2013). No entanto, em condições de sobrecarga como, por exemplo, doenças, acidentes e estresse emocional, pode ocasionar uma condição patológica que requeira assistência - senilidade. Cabe ressaltar que certas alterações decorrentes do processo de senescência podem ter seus efeitos minimizados pela assimilação de um estilo de vida mais ativo.

Envelhecer com qualidade de vida é uma preocupação da área de geriatria e gerontologia, desse modo originou-se formulações teóricas diversas e um extenso discurso a respeito do envelhecimento bem-sucedido, no qual se formulou o conceito de envelhecimento ativo.

Dessa forma, Massi et al. (2018) propõem que o envelhecimento ativo é um termo não se refere à força física de trabalho, mas à participação social do idoso, assinalando para uma nova compreensão acerca da velhice. Nessa concepção, os idosos passam a ser vistos, para além de aspectos meramente orgânicos, podendo ser agentes responsáveis pelas contribuições e benefícios do desenvolvimento social. Para Mattos et al. (2017), o envelhecimento ativo é a forma de envelhecimento almejada para a pessoa idosa, no entanto esse processo depende de um conjunto de fatores relacionados a diversas áreas da vida do indivíduo. Assim, vivenciar essa fase da vida de maneira ativa socialmente e nos demais aspectos da vida se apresenta como um grande desafio a ser encarado pelas atuais políticas de saúde mundial.

Nesse sentido, a prática de atividades lúdicas e educativas possui importância inegável para promover a mudança no estilo de vida de idosos, haja vista que esta tipo de estratégia de educação em saúde permitir que haja momentos de bem-estar físico, mental e de interação interpessoal, podendo propiciar melhora significativa na qualidade de vida de idosos, além do 
Atividades de educação em saúde realizadas com grupo de idosas para promoção do autocuidado em saúde

fortalecimento da autonomia, autoestima, descontração, reflexão e melhor compreensão do tema em discussão (FLEURÍ et al, 2013; PINHEIRO; GOMES, 2014).

Nesse contexto, a Enfermagem exerce um papel singular na prática de estratégias educativas de promoção do envelhecimento saudável. Para tanto, essas atividades devem ser ancoradas na educação em saúde, que proporciona a participação do indivíduo em grupos (MALLMANN et al., 2015). Ressalta-se que a promoção da saúde com enfoque no autocuidado pode contribuir para que os idosos venham a se cuidar, influenciando positivamente na preservação de sua autonomia, independência e condições de saúde. Por isso, faz-se necessário utilizar abordagens que potencializem o empoderamento dos sujeitos para a promoção da autonomia e do autocuidado.

Nessa perspectiva, o referencial teórico-metodológico adotado nas práticas educativas baseou-se nos preceitos da Teoria do Autocuidado de Orem e nas concepções de educação dialógica de Paulo Freire, as quais serviram de suportes para a realização das estratégias educativas desenvolvidas no grupo "Construindo a Melhor Idade".

A teoria de Dorothea Orem afirma que os indivíduos devem ser responsáveis pelo próprio cuidado, porém quando há alguma incapacidade ou agravo à saúde o cuidado deve ser prestado pela Enfermagem. Nesse sentido, Queiroz, Vidinha e Filho (p. 159, 2014) afirmam que “O autocuidado é uma função humana reguladora que as pessoas desempenham deliberadamente por si próprias ou que alguém a execute por eles para preservar a vida, a saúde, o desenvolvimento e o bem-estar".

Para que o autocuidado seja realizado, é necessário que haja uma ampla oferta de ações que promovam a qualidade de vida dos indivíduos resultando no envelhecimento ativo e saudável.

Com vistas a alcançar o propósito traçado para as atividades educativas desenvolvidas junto ao grupo, lançou-se mão dos princípios de educação dialógica de Paulo Freire, os quais constituem importante ferramenta para problematizar e integrar saberes e práticas mediadas pelo diálogo entre sujeitos acadêmicos de enfermagem/docentes e comunidade, favorecendo a interação de educadores e educandos.

Em face das reflexões e dados apresentados no estudo, buscou-se realizar ações que possibilitassem ampliar o acesso das idosas às ações de promoção à saúde e prevenção de doenças. Assim, o estudo objetivou descrever as experiências de atividades de educação em saúde realizadas com idosas tendo como eixos problematizadores o Envelhecimento ativo e o Autocuidado. 
Atividades de educação em saúde realizadas com grupo de idosas para promoção do autocuidado em saúde

\section{MATERIAIS E MÉTODOS}

O presente estudo consiste de um relato de experiência de ações educativas oriundo de um grupo de idosas vinculadas ao 'Projeto de Extensão e Pesquisa em Saúde da Família - PES' desenvolvido por uma IES de Fortaleza-CE. Esse relato destaca situações referentes à prática de educação em saúde promovida por uma docente do curso de Enfermagem (tutora) e acadêmicos de Enfermagem (extensionistas) do PES com idosas, tendo como eixo temático problematizador: o 'Envelhecimento Ativo' e o 'Autocuidado em Saúde'.

As estratégias educativas foram realizadas com as idosas que participam do grupo “Construindo a Melhor Idade", tendo como cenário a clínica-escola de uma IES de Fortaleza que mantém parceria com o Corpo de Bombeiro Militar do Ceará, o qual já desenvolve atividades físicas e de lazer com as referidas participantes. Concernente ao recorte temporal, considerado neste estudo, as ações educativas foram realizadas em 14 encontros, no período de março a dezembro de 2016. Contudo, serão descritas nesse relato as vivências desenvolvidas em quatro encontros, que ocorreram no período de março a junho de 2016.

$\mathrm{Na}$ primeira oficina abordou-se sobre 'Atividade Física na Terceira Idade'. Nesse encontro foram exibidas reportagens sobre a história de alguns idosos que são atletas e esportistas para suscitar reflexões e debates acerca do tema, a fim de incentivar a prática de atividade física entre as participantes. As reportagens apresentadas retratavam diversos idosos que participam de campeonatos como atletas em várias modalidades esportivas, como karatê, boxe, judô, futebol, ciclismo, corrida, entre outras. Estes atribuíam às práticas esportivas, aliada à adoção de bons hábitos de vida, como alimentação saudável, uma considerável melhora na qualidade de vida física, mental e social.

Após a exibição das reportagens abriu-se espaço para troca de experiência entre o grupo, a partir do qual vários assuntos foram trazidos e abordados pelas idosas, como: Quem tem diabetes pode fazer atividade física? Quantos dias por semana se deve praticar atividades físicas? Se não posso ir à acadêmica, como posso fazer atividade física? Quanto tempo de atividade diária para beneficiar a saúde?

O segundo encontro ocorreu inserido no tema "Exercitando o cérebro - quebra cabeças e jogo da memória". Uma das idosas se prontificou a ensinar, para as demais participantes do grupo, exercícios de treinamento de memória que aprendeu em um curso que fez. Logo após, foram demonstradas outras formas de exercitar o cérebro, como por exemplo, com jogo da memória, jogos dos sete erros e quebra-cabeças. 
Atividades de educação em saúde realizadas com grupo de idosas para promoção do autocuidado em saúde

O terceiro recorte temporal dos encontros do projeto abordou sobre o tema "Alimentação saudável, trabalhando a pirâmide nutricional do idoso", na qual se teve como foco a atenção na prevenção de déficit de nutrientes e vitaminas. Para facilitar o entendimento, as participantes construíram uma pirâmide alimentar, baseando-se no conhecimento prévio das idosas sobre os grupos alimentares.

No quarto encontro do grupo, sentiu-se a necessidade de abordar sobre a seguinte temática: 'Cuidados com a pele: tratamento de estética'. O foco do encontro foi o processo de envelhecimento da pele, as mudanças que ocorrem nessa etapa da vida e os cuidados que se devem ter a fim de retardar e/ou prevenir tanto os sinais do envelhecimento como as doenças de pele.

O método vivencial adotado na aplicação das estratégias educativas foi o de oficinas, por se tratar de uma metodologia ativa que favorece a criação de espaços para reflexão e troca de experiências, tornando o lúdico uma ferramenta para facilitar a interação e aprendizagem dos envolvidos nas atividades (SILVA et al., 2013). Optou-se por essa modalidade metodológica pelo fato desta se apresentar como uma proposta de aprendizagem compartilhada que favorece uma quebra da tradicional relação vertical que persiste entre o profissional da saúde e o sujeito da sua ação, sendo uma estratégia facilitadora da expressão individual e coletiva das demandas de vida que influenciam a saúde (LACERDA et al., 2013).

As abordagens metodológicas selecionadas foram desenvolvidas no âmbito dos encontros semanais de educação em saúde com o grupo de idosas, de modo que as ações extensionistas realizadas no projeto envolveram atividades educativas voltadas ao incentivo de práticas saudáveis, favorecendo o envelhecimento ativo e adoção de medidas preventivas de autocuidado em relação à saúde do idoso.

Participaram das oficinas educativas vinte e cinco idosas, com idades entre 60 e 83 anos. No desenvolvimento das práticas educativas, procurou-se adotar uma postura a partir das necessidades reais e dúvidas apresentadas pelas participantes do grupo, adotando-se o diálogo e a troca de conhecimentos e experiências. Desse modo, para nortear os encontros, utilizaram-se as fases propostas por Paulo Freire que fundamentam o Círculo de Cultura (Ciclo Gnosiológico). Essa abordagem permite que os participantes participem ativamente do processo de ensino e de aprendizagem podem pesquisar, pensar, praticar, refletir, sentir, deliberar, ser, plantar, agir, cultivar, intervir e avaliar o seu fazer, num movimento permanentemente dialógico (FREIRE, 2011). Decidiu-se pela metodologia problematizadora de Freire, por acreditar que ela é referência para as práticas educativas em saúde. 
Atividades de educação em saúde realizadas com grupo de idosas para promoção do autocuidado em saúde

Além das concepções de educação de Paulo Freire, utilizou-se também o referencial teórico baseado nos preceitos da Teoria do Autocuidado de Orem (QUEIROS; VIDINHA; FILHO, 2014), que consiste na ideia de que os indivíduos, quando capazes, devem cuidar de si mesmos, com intuito de promover atividades de autocuidado visando o envelhecimento ativo das participantes.

Nesse sentido, Orem desenvolveu uma prática educativa para a saúde, na qual a relação profissional/cliente, aqui extensionistas/idosas, é sempre horizontal, pois se dispõe a mobilizar no cliente a prática do autocuidado, a tomada de decisão para as mudanças necessárias para o autocuidado e a busca de novos conhecimentos/aprendizagens para o enfrentamento das situações de vida e saúde.

Apresenta-se nesse estudo o recorte das atividades desenvolvidas em quatro encontros desse grupo, que se desenvolveram no processo de codificação e decodificação de dois eixos temáticos geradores: o Envelhecimento Ativo e Autocuidado em Saúde. Com intuito de fomentar a livre expressão de ideias das idosas a respeito dos eixos escolhidos, utilizaram-se métodos lúdicos e dinâmicos que favoreceram reflexões acerca das temáticas abordadas em cada encontro semanal, com duração de uma hora.

\section{RESULTADOS E ANÁLISES}

\section{Descrição das atividades educativas}

As temáticas trabalhadas nas oficinas partiram de um momento dialógico dos extensionistas com as idosas que foram: atividade física, exercício do cérebro, alimentação saudável e cuidados com a pele, estas foram as temáticas que foram abordadas com diferentes metodologias ativas.

$\mathrm{Na}$ primeira oficina abordou-se sobre 'Atividade Física na Terceira Idade'. O desenvolvimento dessa atividade em grupo, fundamentada na abordagem do empoderamento, oportunizou a construção de novos conhecimentos a partir da troca de experiências das idosas e participação ativa na aprendizagem, favorecendo uma ressignificação das realidades vivenciadas.

Ademais, acredita-se que as reportagens oportunizaram também a conscientização da importância das idosas adotarem hábitos de vida saudáveis, incluindo a prática de atividade física regular. Nesse sentido, o estudo de Valer et al. (2015) ressalta a importância de englobar nas atividades de promoção da saúde o estímulo ao empoderamento, à capacidade desses idosos se 
Atividades de educação em saúde realizadas com grupo de idosas para promoção do autocuidado em saúde

verem como cidadãos, haja vista que tais práticas podem favorecer a participação ativa nas decisões sobre a sua saúde e sua comunidade.

Com a criação desses espaços de partilha e troca de saberes para se refletir sobre as atividades físicas, precisa-se pensar além dos benefícios biopsicossociais proporcionados pela sua prática, como também, por exemplo, compreender as mudanças de comportamentos individuais e/ou coletivos para a adesão e manutenção dessas atividades. Haja vista que essas só proporcionarão os devidos benefícios, se realizadas de forma contínua e corretamente. Sendo assim, tão importante quanto investigar os benefícios proporcionados por essas práticas, é compreender como motivar as pessoas a se manterem engajadas neste propósito (MACIEL, 2010). Nesse sentido, fez-se necessário os facilitadores discutir com as idosas os fatores que influenciam a adesão das atividades físicas, favorecendo uma mitigação desses fatores.

Observou-se que atividade desenvolvida, a partir das informações em saúde compartilhadas, contribuiu para que as idosas tivessem uma percepção melhor sobre seu autocuidado, implicando em mudanças no estilo de vida, especialmente, a prática de exercícios físicos e a realização de uma dieta balanceada.

A prática regular de atividade física está associada a benefícios à saúde, tais como redução de riscos de doenças cardíacas e cerebrovasculares, diabetes tipo II, hipertensão, câncer de cólon, de mama e depressão, além de ser imprescindível para obtenção do equilíbrio energético e o controle do peso (FARIAS; NACHTIGALL; MYSKIW, 2017). Outrossim, deve-se estimular a população idosa à prática de atividades físicas capazes de promover a melhoria da aptidão física relacionada à saúde.

O segundo encontro abordou a temática "Exercitando o cérebro - quebra cabeças e jogo da memória". Através da realização das dinâmicas propostas, percebeu-se que as idosas foram estimuladas a compartilhar suas dificuldades, nomeando simples atividades corriqueiras e que, no entanto, já não eram mais realizadas de forma satisfatória, as mesmas enfatizaram sobre o conhecimento que tinham a cerca da importância de se praticar atividades como essas, demonstrando, assim, total interação em sua realização. Observou-se também que apesar de alguns erros durante a realização das dinâmicas, estes eram encarados como desafios a serem superados.

Para realização da proposta do encontro, os extensionistas se prontificaram de selecionar diversos jogos de papel, visto que estes podem ser praticados individualmente ou em grupo, além de serem grandes facilitadores da manutenção da memória, sendo inclusive os jogos de palavrascruzadas, os preferidos na indicação dos neurologistas, visto que esses exercícios estimulam a 
Atividades de educação em saúde realizadas com grupo de idosas para promoção do autocuidado em saúde

memória, exigindo que o cérebro faça uma busca em todo o seu conhecimento a cada charada, incitando assim a capacidade de memória e discernimento (SOBACC, 2016).

Em uma pesquisa realizada em 2011, na sede do PUCG/UFF, no Rio de Janeiro, com idosos entre 60 a 85 anos, que realizaram testes de memória, depois participaram de atividades educativas e oficinas sobre memória por um ano, os pesquisadores verificaram que, após frequentarem as oficinas de memória, uma série de atividades passou a ser realizada pelos idosos, dentre as quais, destacam-se: o aumento da frequência de leitura, o número de vezes que saem de casa, a quantidade de amizades feitas e a realização das atividades cognitivas (OLIVEIRA et al., 2012).

Nesse sentido, o intuito maior do desenvolvimento dessa oficina foi o de constituir-se como espaço de socialização desses idosos, oportunizando, ainda, a realização de atividades lúdicas por meio de jogos e exercícios cognitivos a serem realizados no cotidiano de todos.

O terceiro encontro do projeto abordou sobre o tema "Alimentação saudável, trabalhando a pirâmide nutricional do idoso". Antes da construção da pirâmide pelas idosas, discutiu-se em grupo o que é a pirâmide alimentar, mostrando os tipos de alimentos e as porções que precisam ser consumidas diariamente para manter uma alimentação saudável e equilibrada. A respeito da pirâmide, explicou-se que a base larga indica os alimentos imprescindíveis à nutrição e que devem ser consumidos mais frequentemente. Destacou-se também que na medida em que a pirâmide vai estreitando, o consumo de determinados alimentos também deve diminuir, alcançando a ponta da pirâmide, a qual aponta os alimentos que devem ser consumidos em quantidades reduzidas.

Depois das orientações feitas, as idosas confeccionaram a pirâmide alimentar com os recortes trazidos pelos monitores/extensionistas, partindo do esboço da pirâmide em uma cartolina. Incentivou-se que cada participante colasse a figura no local que considerava correto da pirâmide e explicasse por que achava certo. Por conseguinte, ocorria uma recapitulação do que havia sido discutido, porém, de uma forma mais prática, utilizando exemplos de alimentos do diaa-dia.

A partir da realização dessa oficina educativa, constatou-se que o grupo de idosas estava bastante engajado e demonstrava interesse pelo assunto abordado. Embora o tema do encontro seja bastante discutido na mídia, as idosas estavam, durante toda a oficina, fazendo questionamentos e participando das atividades propostas.

Cabe aqui enfatizar que diversos fatores - econômicos e culturais - influenciam diretamente a saúde e a qualidade de vida de idosos. Desse modo, para avaliar e promover a saúde do idoso, diversas variáveis do campo do saber devem ser consideradas de maneira 
Atividades de educação em saúde realizadas com grupo de idosas para promoção do autocuidado em saúde

interdisciplinar e multidimensional. Dentre essas variáveis, a alimentação saudável constitui aspecto importante na qualidade de vida na terceira idade.

Conforme dados do Ministério da Saúde, estima-se que quase 60\% dos idosos brasileiros possuem excesso de peso e cerca de $80 \%$ tem pelo menos uma doença crônica não transmissível (DCNT) (BRASIL, 2013). Além disso, a dieta inadequada aumenta o risco de desenvolvimento de DCNT (LIM et al., 2012). Daí a importância de abordar essa temática por meio de uma metodologia adequada que valorizasse as idosas, levando-se em conta os conhecimentos e saberes que as mesmas já possuíam. Por isso, acredita-se que essa temática deve ser abordada e discutida, avultadamente, com a população idosa, bem como com seus familiares e/ou cuidadores.

Portanto, considerando-se as estratégias de partilha de saberes, Oliveira et al. (2013) considera que as oficinas se constituem como um espaço privilegiado de criação e descobertas cujos produtos construídos apresentam aspectos da contribuição de cada participante. Dessa maneira, a utilização desse tipo de oficina, composta por ações teóricas e práticas, favorece o envolvimento das idosas e, consequentemente, o alcance do objetivo proposto.

No quarto encontro, levando-se em conta as solicitações e sugestões das participantes, foi abordando sobre a seguinte temática: 'Cuidados com a pele: tratamento de estética'. Em parceria com o Curso de Graduação em Estética e Cosmética da IES mentora do Projeto de Extensão e Pesquisa em Saúde da Família - PES, organizou-se um momento para que as idosas recebessem informações sobre como cuidar da pele sem precisar gastar muito dinheiro. Além dessa atividade, as idosas participaram de uma oficina de SPA das mãos, realizada por uma docente do curso de Estética e Cosmética. Com a realização dessa atividade, percebeu-se que, além do estimulo do autocuidado, a prática auxiliou na melhora da autoestima das participantes, haja vista que estas expressaram satisfação diante dos resultados após os procedimentos realizados.

$\mathrm{Na}$ terceira idade, a descamação, principalmente nas extremidades, é bem acentuada, sendo basicamente causada por diminuição da secreção sebácea, por mudanças nos lipídios, associadas a uma menor quantidade de água, que predispõe a pele a maior ressecamento e leva ao aparecimento de fissuras, com resistência diminuída à agressão de substâncias alcalinas, como sabões e outros produtos químicos (FORTES; SUFFREDINI, 2014). Por isso, essas alterações requerem atenção qualificada no cuidado e manutenção da saúde do idoso.

Outro aspecto importante é em relação à estética, haja vista que a beleza e atratividade física são percepção de características físicas que uma pessoa, ou grupo de pessoas, considera bela ou atraente. Tal beleza ou atratividade física pode incluir várias implicações, como, por exemplo, atratividade sexual, graciosidade ou beleza física. Alguns aspectos de como traços físicos são julgados atraentes são universais, enquanto outros são restritos a culturas, sociedades ou períodos 
Atividades de educação em saúde realizadas com grupo de idosas para promoção do autocuidado em saúde

de tempo específicos. A beleza pode ter efeitos significativos em como a pessoa é julgada, em termos de oportunidades sociais, amizades, comportamento sexual, etc. (COPACABANA RUNNERS, 2016).

De modo geral, acredita-se que as estratégias e atividades educativas utilizadas nas oficinas pelos acadêmicos foram potencialmente significativas para o conhecimento das idosas, já que a aprendizagem significativa é individual. É necessário que se faça a relação entre o conteúdo a ser aprendido e o conhecimento prévio das mesmas.

Uma particularidade importante na abordagem das atividades propostas no projeto foi o modo de como se deu a relação dos extensionistas com o grupo, no qual ocorreu de maneira horizontal, sem cobranças, visando a interação e participação mais ativa das idosas nas atividades, mantendo sempre uma postura respeitosa e despida de juízo de valores.

\section{CONSIDERAÇÕES FINAIS}

A experiência descrita assume características próprias devido ao espaço institucional em que se realizou, uma vez que se usufruiu de vantagens da ambiência universitária. Nessa concepção, considera-se que as dinâmicas empregadas na oficina favoreceram um processo educativo-participativo, pois as idosas foram estimuladas a atuar como sujeitos reflexivos e ativos na vivência ensino-aprendizagem, e não como meras expectadoras.

A despeito do que muitos consideram a velhice, ao pensar na redução da funcionalidade, a vida das pessoas "idosas" continua tendo sentido, quando elas permanecem realizando as atividades que lhe proporcione bem-estar físico, psicológico e social. Por isso, é muito importante a participação em atividades grupais que favoreçam conhecimentos para a promoção da saúde, prevenção de doenças, possibilitando interação social, compartilhando saberes e trocas de experiência.

Nessa perspectiva, o processo educativo desenvolvido ocorreu de maneira efetiva entre os facilitadores e as idosas, levando todas a participarem na construção do conhecimento e evitando que somente os extensionistas, de uma maneira unidirecional, ficassem expondo sobre a temática, enquanto que os sujeitos apenas ouvissem essas informações sem manifestar suas dúvidas.

Ressalta-se ainda que a parceria entre ensino, pesquisa e extensão e a aproximação da academia com a comunidade legitima o lócus acadêmico como espaço de produção de conhecimento científico para pensar e repensar as práticas de saúde, aberto à inserção de propostas inovadoras. 
Atividades de educação em saúde realizadas com grupo de idosas para promoção do autocuidado em saúde

Em síntese, considerou-se exitosa a utilização das oficinas educativas como estratégia de Educação em Saúde, pois percebeu-se que as idosas ficaram mais acessíveis e à vontade para expor suas dúvidas. Por meio dessa estratégia, as participantes relataram sentir-se motivadas, o que é imprescindível, visto que que no processo de Educação em Saúde é necessário, primeiro, que haja a automotivação para que seja possível a construção do conhecimento, tendo como objetivo promover a adoção de hábitos saudáveis.

\section{AGRADECIMENTOS}

Às idosas do grupo "Construindo a Melhor idade" integrantes do Projeto de Extensão e Pesquisa em Saúde da Família - PES, que nos proporcionaram uma experiência quotidiana de profundo contato afetivo, trocas de saberes e de aprendizado de vida, e também a todos os docentes e acadêmicos que atuaram no projeto, por compartilharem e tornarem possível esta construção.

\section{REFERÊNCIAS}

BRASIL. Ministério da Saúde. Vigitel Brasil 2012: Vigilância de Fatores de Risco e Proteção para Doenças Crônicas por Inquérito Telefônico. Brasília: Ministério da Saúde, 2013.

COPACABANA RUNNERS. Beleza. Disponível em: <http://www.copacabanarunners.net/beleza.html>. Acesso em: 7 mar. 2017.

DAWALIBI, N. W.; ANACLETO, G. M. C,; WITTER, C.; GOULART, R. M. M.; AQUINO, R. C. Envelhecimento e qualidade de vida: análise da produção científica da SciEL. Estudos de Psicologia, Campinas, v. 30, n. 3, p. 393-403, jul./set. 2013.

FACULDADE METROPOLITANA DA GRANDE FORTALEZA (FAMETRO). Resolução no .19 de 27 de setembro de 2012 do CEPEX. Fortaleza: FAMETRO, 2012.

FARIAS, C. P.; NACHTIGALL, E. G.; MYSKIW, J. C. Atividade física exerce papel protetor no cérebro. In: TERRA, N. L. et al. (Orgs.). Temas de geriatria e gerontologia. Porto Alegre: EDIPUCRS, 2017.

FLEURÍ, A. C. P. et al. Atividades lúdicas com idosos institucionalizados. Enfermagem Revista, Belo Horizonte, v. 16, n. 1, p. 50-57, 2013.

FORTES, T. M. L.; SUFFREDINI, I. B. Avaliação de pele em idoso: revisão da literatura. J Health Sci Inst.; v. 32, n. 1, p. 94-101, 2014.

FREIRE, P. Educação como prática de liberdade. Rio de Janeiro: Paz e Terra, 2011. 
Atividades de educação em saúde realizadas com grupo de idosas para promoção do autocuidado em saúde

INSTITUTO BRASILEIRO DE GEOGRAFIA E ESTATÍSTICA (IBGE). Mudanças demográficas no Brasil no início do século XXI. Subsídios para as projeções da população. Brasília: IBGE, 2016.

Pesquisa Nacional por Amostra de Domicílios: síntese de indicadores 2014. Rio de Janeiro: IBGE, 2015.

LACERDA, A. B. M. et al. Oficinas educativas como estratégia de promoção da saúde auditiva do adolescente: estudo exploratório. Audiol., Commun. Res., São Paulo, v. 18, n. 2, p. 85-92, jun. 2013. Disponível em: <http://www.scielo.br/scielo.php?script=sci arttext\&pid=S231764312013000200006\&lng=en\&nrm=iso > . Acesso em: 14 fev. 2018.

LIM, S. S.; VOS, T.; FLAXMAN, A. D.; DANAEI, G.; SHIBUYA, K.; ADAIR-ROHANI, H. et al. A comparative risk assessment of burden of disease and injury attributable to 67 risk factors and risk factor clusters in 21 regions, 1990-2010: a systematic analysis for the Global Burden of Disease Study 2010. Lancet, v. 380, n. 9859, p. 2224-2260, 2012.

MACIEL, M. G. Atividade física e funcionalidade do idoso. Motriz. Rio Claro, v.16 n.4, p.10241032, out./dez. 2010.

MALLMANN, D. G.; NETO, N. M. G.; SOUSA, J. C.; VASCONCELOS, E. M. R. Educação em saúde como principal alternativa para promover a saúde do idoso. Ciência \& Saúde Coletiva, v. 20, n. 6, p. 1763-1772, 2015.

MASSI, G. et al. Active aging: an intervention-research report. Rev. CEFAC, São Paulo, v. 20, n. 1, p. 5-12, fev. 2018. Disponível em:

$<\underline{\text { http: } / / \text { www.scielo.br/scielo.php?script }=\text { sci arttext\&pid }=S 1516-}$

18462018000100005\&lng=en\&nrm=iso > . Acesso em: 04 mar. 2018.

MATTOS, C. M. Z.; MOTA, K. M. S.; GEHLEN, M. H.; GROSSI, P. K. Envelhecimento ativo: uma realidade distante para pessoas idosas em situação de rua. In: TERRA, N. L. et al. (Orgs.). Temas de geriatria e gerontologia. Porto Alegre: EDIPUCRS, 2017.

OLIVEIRA, C. S.; COSTA, S. R.; SANTOS, I. C.; LEMOS, C. E. Oficina de memória para idosos: espaço para conhecimento, socialização e ludicidade. RBCEH. Passo Fundo, v. 9, n. 2, p. 180-192, maio/ago. 2012.

OLIVEIRA, R. G.; FROTA, N. M.; MAGALHÃES, S. R.; BARROS, L. M. Oficinas educativas para promoção da saúde de idosas institucionalizadas. Rev Bras Promoc Saude, Fortaleza, v. 26, n. 4, p. 595-600, out./dez., 2013.

PINHEIRO, S. B.; GOMES, M. L. Efeitos das atividades lúdicas no idoso com alteração do cognitivo leve. Revista Pesquisa em Fisioterapia, Salvador, v. 4, n. 1, p. 71-77, 2014.

QUEIROS, P. J. P.; VIDINHA, T. S. S.; FILHO, A. J. A. Autocuidado: o contributo teórico de Orem para a disciplina e profissão de Enfermagem. Rev. Enf. Ref. Coimbra, v. ser IV, n. 3, p. 157-164, dez. 2014. Disponível em: $\leq$ http://www.scielo.mec.pt/scielo.php?script=sci arttext\&pid=S087402832014000300018\&lng=pt\&nrm=iso $>$. Acesso em: 09 mar. 2018.

SCHOTT, M. Integração ensino-serviço-comunidade na educação em saúde: desafios e potencialidades. Alagoas: $2016 . \quad$ Disponível em: 
Atividades de educação em saúde realizadas com grupo de idosas para promoção do autocuidado em saúde

< http://www.convibra.com.br/upload/paper/2016/58/2016 58 12936.pdf $>$. Acesso em: 02 mar. 2018.

SILVA, D. M.; ALVES, M. R.; SOUZA, T. O.; DUARTE, A. C. S. Sexualidade na adolescência: relato de experiência. J Nurs UFPE on line., v. 7, n.1, p. 820-832, 2013. Disponível em: $<$ http://www.revista.ufpe.br/revistaenfermagem/index.php/revista/article/viewFile/3681/p df 2201>. Acesso em: 02 mar. 2018.

SOCIEDADE BRASILEIRA DE ARTE, CULTURA E CIDADANIA (SOBACC). Exercitando o cérebro: jogos para a terceira idade. Fortaleza: SOBACC, 2016.

VALCARENGHI, R. V.; LOURENCO, L. F. L.; SIEWERT, J. S.; ALVAREZ, A. M. Nursing scientific production on health promotion, chronic condition, and aging. Rev. Bras. Enferm. Brasília, v. 68, n. 4, p. 705-712, Aug. 2015.

VALER, D. B. et al. The significance of healthy aging for older persons who participated in health education groups. Rev. bras. geriatr. gerontol., Rio de Janeiro, v. 18, n. 4, p. 809-819, dez. 2015. Disponível em: <http://www.scielo.br/scielo.php?script=sci arttext\&pid=S180998232015000400809\&lng=en\&nrm=iso > . Acesso em: 04 mar. 2018. 